\title{
Probabilistic risk analysis of groundwater remediation strategies
}

\author{
D. Bolster, ${ }^{1}$ M. Barahona, ${ }^{1}$ M. Dentz, ${ }^{1}$ D. Fernandez-Garcia, ${ }^{1}$ X. Sanchez-Vila, ${ }^{1}$ \\ P. Trinchero, ${ }^{1}$ C. Valhondo, ${ }^{1}$ and D. M. Tartakovsky ${ }^{2}$ \\ Received 28 October 2008; revised 21 February 2009; accepted 11 March 2009; published 12 June 2009.
}

[1] Heterogeneity of subsurface environments and insufficient site characterization are some of the reasons why decisions about groundwater exploitation and remediation have to be made under uncertainty. A typical decision maker chooses between several alternative remediation strategies by balancing their respective costs with the probability of their success or failure. We conduct a probabilistic risk assessment (PRA) to determine the likelihood of the success of a permeable reactive barrier, one of the leading approaches to groundwater remediation. While PRA is used extensively in many engineering fields, its applications in hydrogeology are scarce. This is because rigorous PRA requires one to quantify structural and parametric uncertainties inherent in predictions of subsurface flow and transport. We demonstrate how PRA can facilitate a comprehensive uncertainty quantification for complex subsurface phenomena by identifying key transport processes contributing to a barrier's failure, each of which is amenable to uncertainty analysis. Probability of failure of a remediation strategy is computed by combining independent and conditional probabilities of failure of each process. Individual probabilities can be evaluated either analytically or numerically or, barring both, can be inferred from expert opinion.

Citation: Bolster, D., M. Barahona, M. Dentz, D. Fernandez-Garcia, X. Sanchez-Vila, P. Trinchero, C. Valhondo, and D. M. Tartakovsky (2009), Probabilistic risk analysis of groundwater remediation strategies, Water Resour. Res., 45, W06413, doi:10.1029/2008WR007551.

\section{Introduction}

[2] Heterogeneity, the ubiquitous lack of complete site characterization and conceptual-mathematical limitations of many modeling approaches are some of the reasons rendering deterministic predictions of subsurface flow and transport suboptimal. Quantification of these and other sources of uncertainty is a prerequisite for modern, scientifically defensible decision making in the areas of groundwater exploitation and remediation. Many pressing problems, such as the selection of a remediation strategy most likely to succeed at a given contaminated site or the assessment of the likelihood that supercritical carbon dioxide sequestered in the subsurface escapes back into the atmosphere, cannot be reliably answered without proper uncertainty quantification and risk analysis. When these modeling components are ignored, the failure of engineering campaigns to control the fate and migration of contaminants is common, as is exemplified by the frequency with which contaminant plumes bypass permeable reactive barriers constructed at locations suggested by deterministic analyses (Interstate Technology and Regulatory Council (ITCR),

\footnotetext{
${ }^{1}$ Department of Geotechnical Engineering and Geosciences, Technical University of Catalonia, Barcelona, Spain.

${ }^{2}$ Department of Mechanical and Aerospace Engineering, University of California, San Diego, La Jolla, California, USA.

Copyright 2009 by the American Geophysical Union. 0043-1397/09/2008WR007551\$09.00
}

Permeable reactive barriers: Lessons learned/new directions, 2005, available at www.itrcweb.org).

[3] While a consensus is emerging that risk analysis must be an integral part of decision making in subsurface hydrology, its precise operational definition is still a subject of debate. Do a few realizations of Monte Carlo simulations and/or a sensitivity analysis constitute a risk assessment? Scientific disciplines where risks are routinely evaluated to satisfy statutory requirements, e.g., nuclear power generation [National Research Council (NRC), 1983], aerospace industry [Paté-Cornell and Dillon, 2001], and earthquake engineering [NRC, 1997], provide some guidance. Specifically, a comprehensive risk analysis can be defined as a procedure that enables one to answer the following three questions: What can happen? How likely is it to happen? Given that it occurs, what are the consequences? [Bedford and Cooke, 2003]. The National Research Council's report $[N R C, 1997]$ on seismic hazard analysis explicitly identifies the following attributes of risk assessment (RA): (1) RA must be probabilistic and quantitative; (2) RA must be based on subjective probabilities; and (3) The main focus of any probabilistic RA (PRA) must be on uncertainty quantification.

[4] The reasoning behind the report is equally applicable to subsurface hydrology. The emphasis on the quantitative (as opposed to descriptive or qualitative) nature of PRA is important. The realization that a statement "the contamination is likely to occur" is insufficient, unless accompanied by a probability value of such an occurrence, can be traced back to Garrick [1989] and is a fundamental tenant of modern PRA [Bedford and Cooke, 2003]. Equally important is the 


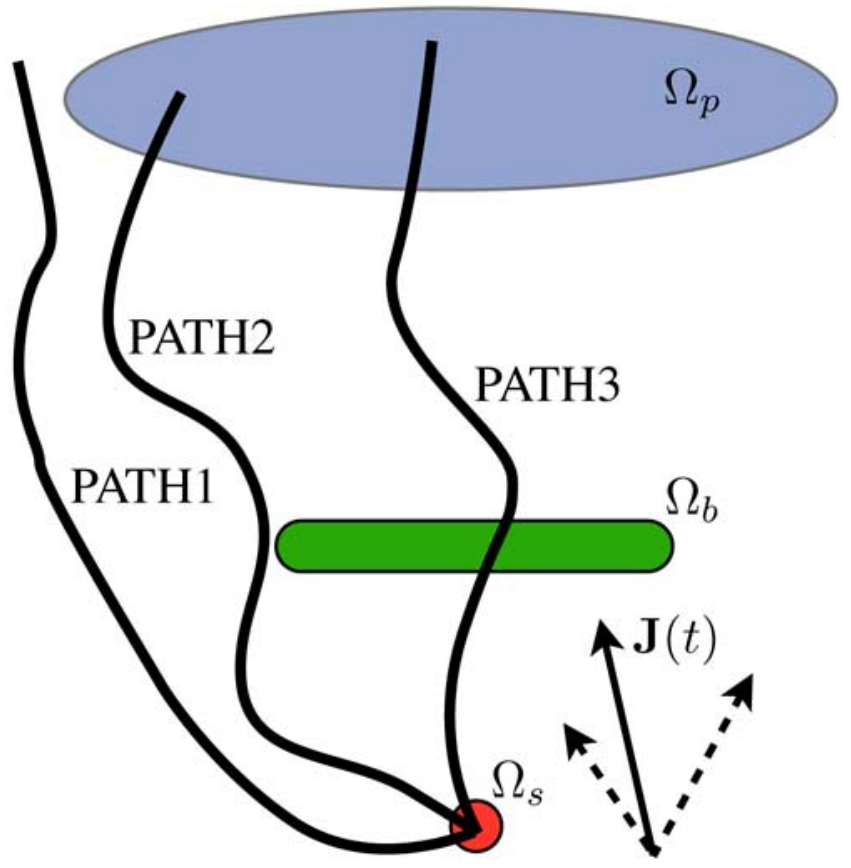

Figure 1. A schematic representation of the aquifer remediation problem. A potential source of contamination is located at $\Omega_{s}$. Driven by hydraulic gradient $\mathbf{J}(t)$, a contaminant plume migrates toward region $\Omega_{p}$, which one aims to protect by constructing a permeable reactive barrier $\Omega_{b}$. Three alternative paths of the plume migration are denoted by PATH 1, PATH 2, and PATH 3.

use of subjective probabilities. Since subjective probability $P(A)$ is defined as "a degree of belief, of one individual, in the occurrence of $A$ " [Bedford and Cooke, 2003, p. 24], it paves the way for incorporation of expert knowledge and other soft data into the modeling process [NRC, 1997].

[5] A rigorous PRA framework lays out a formal procedure for risk analysis, which is implemented in this study. The procedure consists of [Vesely et al., 1981; Bedford and Cooke, 2003] (1) identification of key components/events (a contaminant's release, failure of a permeable reactive barrier to intercept the plume, etc.) contributing to a system's failure (aquifer contamination, escape of the sequestered $\mathrm{CO}_{2}$ into the atmosphere, etc.); (2) construction of a fault tree or a binary decision diagram; (3) their mathematical representation by means of Boolean algebra, which allows one to relate the probability of a system's failure to that of its critical constitutive components; and (4) computation of the latter probabilities.

[6] Applications of this PRA procedure to distributed physical systems include work by Tartakovsky [2007], Winter and Tartakovsky [2008], and Bolster and Tartakovsky [2008]. (Less formal approaches to PRA in hydrogeology have also been reported [e.g., Batchelor et al., 1998; Wang and McTernan, 2002].) PRA of complex physical phenomena, including subsurface flow and transport, poses a set of challenges not routinely encountered in PRA of artificial (engineered) systems. Chief among them is the estimation of the probabilities of failure of individual components, i.e., the probabilities of the occurrence of each event. While in manufactured systems (e.g., space shuttles or nuclear power plants discussed above) such probabilities can often be found in reliability databases, in hydrogeology they have to be either computed by solving flow and transport equations with uncertain parameters or inferred from expert knowledge.

[7] The former option relies on Monte Carlo simulations or other computational approaches, including moment equations, probability density function (pdf) methods, stochastic collocation methods, and stochastic finite elements. The second option is based on prior experience, site's geology, etc. The formal PRA procedure facilitates both approaches by replacing an intractable problem of risk assessment for a subsurface system with hundreds of uncertain parameters with key subsystems, each of which can be characterized by only a few uncertain parameters. When the probability of failure of a given subsystem cannot be evaluated exactly, it might be possible to compute its upper bound that provides a conservative estimate of risk. These concepts are explored in detail below.

[8] In section 2 we formulate a problem of selecting an optimal remediation strategy by using the probability of its success as a criterion. (Costs associated with alternative remediation strategies, another important selection criterion, are not considered here. They can be accounted for in a cost-benefit analysis for which risk assessment provides input.) We present a rigorous PRA of one popular remediation strategy, a permeable reactive barrier; probabilities of success/failure of other remediation strategies can be evaluated in a similar fashion. Section 3 presents a fault tree analysis of a typical permeable reactive barrier. Probabilities of the occurrence of basic events in this tree are evaluated in section 4 . These are combined to compute the probability of failure of a permeable reactive barrier in section 5. Section 6 contains a sensitivity analysis that allows one to determine the sources of uncertainty that contribute most to the possible remediation failure and hence need to be alleviated by collecting more data.

\section{Problem Formulation}

[9] Consider a contaminant plume traveling from a (point or distributed) contamination source $\Omega_{s}$ toward a region $\Omega_{p}$ that has to be protected from contamination (Figure 1). The protected region $\Omega_{p}$ represents a river, municipal wells, etc; $\Omega_{s}$ represents either an actual or potential source of contamination. The plume is driven by a field-scale hydraulic head gradient $\mathbf{J}(t)$, whose temporal variability is caused by seasonal fluctuations in precipitation, seasonal variations in groundwater pumping, artificial groundwater recharge, etc. In field-scale applications, $\mathbf{J}(t)$ is routinely inferred from water level data, e.g., by means of three-point estimators [Silliman and Frost, 1998]. Alternatively, it can be computed by solving flow equations with uncertain hydraulic conductivity, boundary conditions, etc.

[10] Our goal is to select a remediation strategy that would prevent contamination of $\Omega_{p}$. Typical selection criteria are the construction, operation and maintenance costs and the likelihood of success or, equivalently, the probability of failure. In this study, we concentrate on the latter aspect of risk assessment applied to a permeable reactive barrier $\left(\Omega_{b}\right.$ in Figure 1) and natural attenuation; other remediation strategies can be analyzed in a similar manner.

[11] Contaminant migration toward $\Omega_{p}$ can take one of the three paths shown in Figure 1, either bypassing the permeable 
Table 1. Glossary of Event Abbreviations

\begin{tabular}{lc}
\hline \multicolumn{1}{c}{ Event } & Abbreviation \\
\hline System failure & SF \\
Spill occurs & SO \\
Path 1 & P1 \\
Path 2 & P2 \\
Natural attenuation fails & NA \\
Path 3 & P3 \\
Remediation effort fails & RE \\
\hline
\end{tabular}

reactive barrier or being intercepted by it. It is in acknowledging the possibility of each of these scenarios that uncertainty quantification becomes an indispensable component, or the main focus, of risk assessment. If the hydraulic head gradient $\mathbf{J}$ and the aquifer's hydraulic and transport properties were all known with certainty, one would not talk about risk: a properly constructed reactive barrier would accomplish its task, as similar barriers do in a controlled laboratory setting. In actual applications, estimates and future predictions of $\mathbf{J}$ are subject to error, aquifers are heterogeneous, and site-specific data are scarce. These and other sources of uncertainty have to be quantified if one were to have any notion of the likelihood of the success of a remediation strategy.

[12] Depending on the application, various definitions of success or failure are possible. We say that a remediation effort failed if a contaminant's aqueous concentration $C(\mathbf{x}, t)$ at any point $\mathbf{x} \in \Omega_{p}$ exceeds some prespecified value $C^{*}$ within a legally mandated time interval $t \leq T$. The value of $C^{*}$ is typically determined by environmental regulations, for example as a variable in the excess lifetime cancer risk (ELCR) [U.S. Environmental Protection Agency, 1992]. Other (uncertain) variables in ELCR characterize water use (ingestion, inhalation, etc.) and population (age, body weight, etc.) [Tartakovsky, 2007]. Analysis of these factors lies outside the scope of the present analysis, but can be readily accounted for if relevant statistics are available.

[13] To sum up, we formulate the problem of risk assessment for a permeable reactive barrier as follows. Given measurements of hydraulic and transport properties of an aquifer and field-scale hydraulic head gradient, determine the probability of failure of a reactive barrier to prevent contaminant's aqueous concentration $C(\mathbf{x}, t)$ from exceeding the mandated concentration $C^{*}$ for all $\mathbf{x} \in \Omega_{p}$ and $t \leq T$.

[14] It is worthwhile noting that this problem is the opposite of another remediation problem in which $\Omega_{p}$ represents a catchment zone of a pump-and-treat remediation effort. In this case, one would estimate the risk of a contaminant plume bypassing $\Omega_{p}$. The results of the analysis below are equally applicable to this problem, after $\Omega_{b}$ and the corresponding calculations are eliminated.

\section{Fault Tree Analysis}

[15] Risk analysis starts with the identification of basic events that can lead to the system failure (SF), i.e., to the aqueous concentration $C(\mathbf{x}, t)$ exceeding the mandated concentration $C^{*}$ for all $\mathbf{x} \in \Omega_{p}$ and $t \leq T$. The basic events of relevance to our analysis are listed in Table 1 . The initiating event is the occurrence of a spill or multiple spills
(SO). One can be uncertain about this event for a variety of reasons: How likely is a spill to occur? If it has already occurred, what its total mass and duration? Etc. Subsequent events are determined by possible paths of contaminant migration. Event $P 3$ occurs if the contaminant plume is intercepted by the permeable reactive barrier (PATH 3 in Figure 1). Event $P 2$ occurs if the plume bypasses the reactive barrier $\Omega_{b}$ and enters the protected zone $\Omega_{p}$ (PATH 2 in Figure 1). Event $P 1$ is associated with PATH 1 , in which the plume bypasses both the reactive barrier $\Omega_{b}$ and the protected zone $\Omega_{p}$. It does not lead to system failure, since it cannot change contaminant concentration in $\Omega_{p}$. (This reasoning highlights the importance of unambiguous definition of system failure. Alternatively, one could say that the system failed if concentration $C$ at any point in the aquifer exceeds a threshold concentration $C^{*}$ or if the contaminant retains the ability to reach $\Omega_{p}$ at times $t>T$. The contribution of event $P 1$ to the system failure thus defined would have a finite probability.) Event $P 1$ is clearly a reciprocal of the events $P 2$ and $P 3$ in the sense that the probability of its occurrence, $P[P 1]=1-P[P 2]-P[P 3]$ is completely determined by $P[P 2]$ and $P[P 3]$ the probabilities of occurrence of events $P 2$ and $P 3$, respectively. For this reason, event $P 1$ is not explicitly included in the risk assessment.

[16] Contaminant migration along either PATH 2 or PATH 3 does not necessarily lead to system failure. First, natural attenuation, which we define as "the combination of natural biological, chemical, and physical processes that act without human intervention to reduce the mass, toxicity, mobility, or concentration of the contaminants" [Alvarez and Illman, 2006, p. 569], can reduce the contaminant concentration $C$ to levels below $C^{*}$ by the time the plume reaches $\Omega_{p}$. Event $N A$ corresponds to the failure of natural attenuation to achieve this reduction within a time interval $t \in[0, T]$. Finally, if the contaminant plume travels along PATH 3 we must account for event $R E$, the possibility that the reactive barrier $\Omega_{b}$ fails to achieve the required reduction of the contaminant concentration $C$. In this scenario, natural attenuation acts in conjunction with the remediation effort.

[17] The next step in PRA is to construct a fault tree [Bedford and Cooke, 2003; Tartakovsky, 2007], which relates system failure to the occurrence of the basic events identified above. The fault tree for the problem under consideration is shown in Figure 2. The Boolean operators AND and OR indicate a collection of basic events that would lead to failure. The fault tree in Figure 2 enables one to identify the minimal cut sets, the smallest collections of events that must occur jointly in order for the system to fail. In our case, there are two such sets: $\{S O$, $P 2, N A\}$ and $\{S O, P 3, N A, R E\}$. Finally, we use these minimal cut sets to represent the fault tree in terms of the Boolean operators,

$$
S F=S O_{\mathrm{AND}}\left[\left(P 2_{\mathrm{AND}} N A\right) \mathrm{OR}\left(P 3_{\mathrm{AND}} N A_{\mathrm{AND}} R E\right)\right] .
$$

[18] Since $S O$ is independent of the other events, it follows from (1) that the probability of system failure is given by

$$
P[S F]=P[P 2 \cap N A] P[S O]+P[P 3 \cap R E \cap N A] P[S O] .
$$




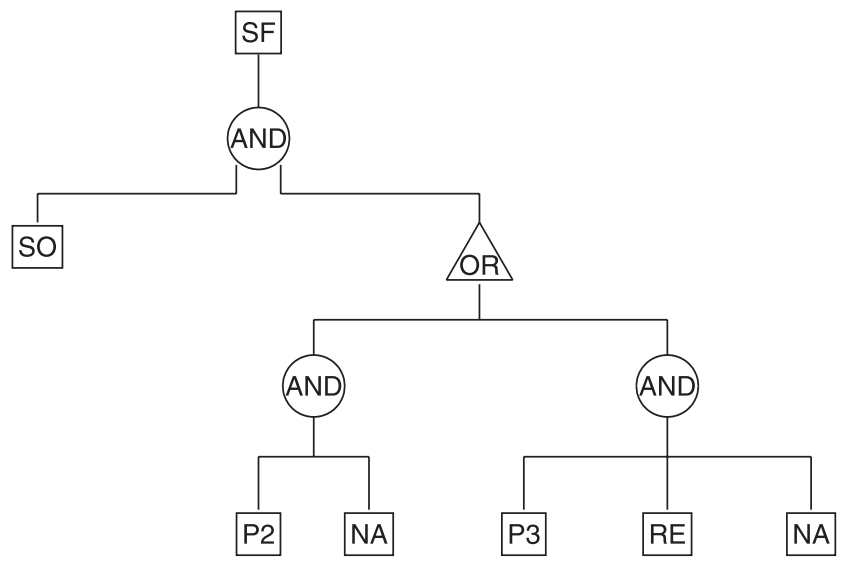

Figure 2. Fault tree for remediation effort.

where $X \cap Y=X$ AND $Y$ and $X \cup Y=X$ OR $Y$. It remains to compute the probabilities of the basic events in (2).

\section{Probabilities of Basic Events}

[19] The fault tree analysis resulting in (2) allows one to replace an intractable task of computing $P[S F]$ as a solution of flow and (reactive) transport equations with a large number of uncertain parameters, with a manageable task of identifying the probabilities of basic events. Many of these events have been analyzed earlier in other contexts. Others are specific enough to be amenable to an expert analysis based on previous experience. Both approaches are combined below for the complete evaluation of $P[S F]$.

\subsection{Probability of PATH 2}

[20] We define $P[P 2]$ as the probability that the plume's center of mass $\mathbf{m}=\left(m_{x}, m_{y}\right)^{T}$ reaches the protected zone $\Omega_{p}$ without ever having passed through the reactive barrier $\Omega_{b}$,

$$
P[P 2] \equiv P\left[\mathbf{m}\left(t^{\prime}\right) \notin \Omega_{b} ; \mathbf{m}(t) \in \Omega_{p} ; 0<t^{\prime}<t<T\right] .
$$

[21] To simplify the presentation and without any loss of generality, we take the reactive barrier $\Omega_{b}$ to be parallel to the protected zone $\Omega_{p}$ and align them with the $x$ axis (Figure 3 ). The source $\Omega_{s}$ is reduced to a point and located at $(x, y)=(0,0)$. Furthermore, we assume that the thickness in the $x$ direction is small compared to the extension in the $y$ direction. Then the domains $\Omega_{i}(i=b, p)$ are represented by the collections of points with coordinates $x_{i}=L_{i}$ and $y_{i}$ $\in\left[y_{i \min }, y_{i \max }\right] ; L_{b}$ and $L_{p}$ denote the distances from the source of contamination $\Omega_{s}$ to the reactive barrier $\Omega_{b}$ and the protected zone $\Omega_{p}$, respectively; $t_{b}$ is the travel time of the plume's center of mass between $x=0$ and $x=L_{b}, t_{p}$ is the travel time from $x=L_{b}$ to $x=L_{p}$. This allows us to replace $(3 a)$ with

$$
P[P 2]=P\left[m_{y}\left(t_{b}\right) \notin \Omega_{b} ; m_{y}\left(t_{b}+t_{p}\right) \in \Omega_{p} ; 0<t_{p}+t_{b}<T\right] .
$$

[22] Let $p_{12}\left(y_{p}, t_{p}+t_{b} ; y_{b}, t_{b}\right)=p_{2}\left(y_{p}, t_{p}+t_{b} \mid y_{1}, t_{b}\right) p_{1}\left(y_{b}, t_{b}\right)$ denote the joint pdf describing the random event $\left\{m_{y}\left(t_{b}\right)=y_{b}\right.$; $\left.m_{y}\left(t_{p}+t_{b}\right)=y_{p}\right\}$, where $p_{12}\left(y_{p}, t_{p}+t_{b} \mid y_{b}, t_{b}\right)$ is the pdf for the event $\left\{m_{y}\left(t_{p}+t_{b}\right)=y_{p}\right\}$ conditioned on the occurrence of the event $\left\{m_{y}\left(t_{b}\right)=y_{b}\right\}$ and $p_{1}\left(y_{b}, t_{b}\right)$ is the pdf for the event $\left\{m_{y}\left(t_{b}\right)=y_{b}\right\}$; the joint distribution of travel times $t_{b}$ and $t_{p}$ is denoted by $f_{p b}\left(t_{p}, t_{b}\right)$. Note that $y_{b}$ and $y_{p}$ are points in the planes $x=L_{b}$ and $x=L_{p}$, respectively, because $t_{b}$ and $t_{p}$ denote the travel times to these planes. Now we can write down the joint probability $P_{12}\left(y_{p} \in \Omega_{p}, t_{p}+t_{b} ; y_{b} \in \bar{\Omega}_{b}, t_{b}\right)$ that $y_{p} \in \Omega_{p}$ and $y_{b} \in \bar{\Omega}_{b}$,

$$
\begin{aligned}
& P_{12}\left(y_{p} \in \Omega_{p}, t_{p}+t_{b} ; y_{b} \in \bar{\Omega}_{b}, t_{b}\right) \\
& =\int_{\Omega_{p}} \int_{\bar{\Omega}_{b}} p_{12}\left(y_{p}, t_{p}+t_{b} ; y_{b}, t_{b}\right) \mathrm{d} y_{b} \mathrm{~d} y_{p} \\
& =\int_{\Omega_{p}} \int_{\bar{\Omega}_{b}} p_{2}\left(y_{p}, t_{p}+t_{b} \mid y_{b}, t_{b}\right) p_{1}\left(y_{b}, t_{b}\right) \mathrm{d} y_{b} \mathrm{~d} y_{p},
\end{aligned}
$$

where $\bar{\Omega}_{b}$ is the complement of $\Omega_{b}$. Integration over the travel times then gives $P[P 2]$ as

$$
\begin{aligned}
P[P 2]= & \int_{0}^{T} \int_{0}^{T-t_{p}} f_{p b}\left(t_{p}, t_{b}\right) \\
& \times P_{12}\left(y_{p} \in \Omega_{p}, t_{p}+t_{b} ; y_{b} \in \bar{\Omega}_{b}, t_{b}\right) \mathrm{d} t_{b} \mathrm{~d} t_{p} .
\end{aligned}
$$

[23] The unknown pdf's $p_{1}$ and $p_{2}$ encapsulate uncertainty about the locations at which the center of mass crosses the planes $x=L_{b}$ and $x=L_{p}$, respectively. In the absence of site-specific data and/or as a prior, one can select these distributions to be Gaussian. A choice of prior variances reflects one's expectation about the magnitude of the plume's deviation from the direction of mean flow (Figure 3). Information about site geology, local transmissivity, hydraulic head, contaminant concentration, etc can then be used to compute the posterior pdf's $p_{1}$ and $p_{2}$ through various parametric inference strategies.

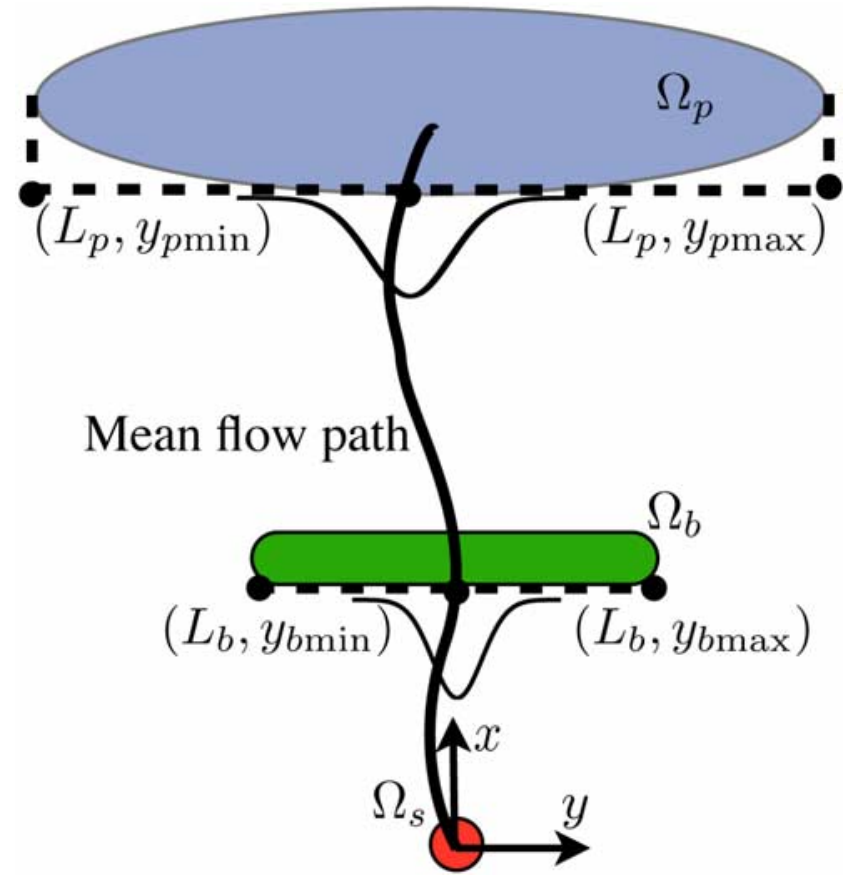

Figure 3. Coordinate system and probability density functions (pdf's) of the center of mass at $x=L_{b}$ and $x=L_{p}$. 
[24] The analysis of Dentz and Carrera [2003] supports the notion that pdf's $p_{1}$ and $p_{2}$ might indeed be Gaussian. In the following, we treat the hydraulic head gradient $\mathbf{J}(t)$ as the only source of uncertainty (modeling it as a Gaussian random process). We focus on times larger than the correlation time of $\mathbf{J}(t)$, denoted by $\tau_{v}$, so that the process $\mathbf{m}(t)$ can be assumed Markovian. This is done so that the path of the plume can be split into two distinct parts without history effects (i.e., the trajectory of the plume from the plane in which the barrier lies to the protection zone, is independent of the trajectory from the spill zone to the barrier plane). According to the analysis of Dentz and Carrera [2003], $p_{1}\left(y_{b}, t_{b}\right)$ and $p_{12}\left(y_{p}, t_{p}+t_{b} \mid y_{b}, t_{b}\right)$ are given by

$$
\begin{aligned}
p_{1}\left(y_{b}, t_{b}\right) & =\frac{1}{\sqrt{4 \pi D^{c m} t_{b}}} \exp \left[-\frac{y_{b}^{2}}{4 D^{c m} t_{b}}\right] \\
p\left(y_{p}, t_{p}+t_{b} \mid y_{b}, t_{b}\right) & =\frac{1}{\sqrt{2 \pi} D^{c m} t_{p}} \exp \left[-\frac{\left(y_{p}-y_{b}\right)^{2}}{4 D^{c m} t_{p}}\right],
\end{aligned}
$$

where

$$
D^{c m}=D^{\text {local }}+\bar{v}^{2} \sigma_{v}^{2} \tau_{v}
$$

[25] The dispersion coefficient $D^{c m}$ provides a measure of the temporal increase of uncertainty in the center of mass position due to fluctuations of the head gradient that adds to the local dispersion coefficient $D^{\text {local }}$ (as the center of mass of the plume does not diffuse, this is zero); $\bar{v}$ is the ensemble mean velocity, $\sigma_{v}^{2}$ the variance of the gradient fluctuations. Travel time $t_{b}$ is the time it takes the plume to migrate from the source of contamination to $\left(x_{b}, y_{b}\right)$. It can either be approximated by the mean travel time $t_{b} \approx L_{b} / \bar{v}$ or treated as a random variable whose statistics are coupled to trajectory moments [Sanchez-Vila and Guadagnini, 2005; Riva et al., 2006].

[26] Note that because of the Markovianity of the underlying random process, the joint distribution of travel times, $f_{p b}\left(t_{p}, t_{b}\right)$ factorizes according to

$$
f_{p b}\left(t_{p}, t_{b}\right)=f\left(L_{p}, t_{p}\right) f\left(L_{b}, t_{b}\right)
$$

[27] Here we can determine the travel time distributions explicitly. The distribution of travel times to reach a control plane at $x=L$ is given by

$$
f(t, L)=\frac{L+\bar{v} t}{2 \sqrt{4 \pi D^{c m} t^{3}}} \exp \left[-\frac{(L-\bar{v} t)^{2}}{4 D^{c m} t}\right] .
$$

The cumulative travel time distribution is given by

$$
F(t, L)=\int_{0}^{t} f\left(t^{\prime}, L\right) \mathrm{d} t^{\prime}=\frac{1}{2}+\frac{1}{2} \operatorname{erf}\left[\frac{\bar{v} t-L}{\sqrt{4 D^{c m} t}}\right] .
$$

The travel time mean and variance are given by

$$
\tau_{L}=\frac{L}{\bar{v}}+\frac{D^{c m}}{\bar{v}^{2}}, \quad \sigma_{\tau_{L}}^{2}=2 \frac{L}{\bar{v}} \frac{D^{c m}}{\bar{v}^{2}}+5 \frac{D^{c m}}{\bar{v}^{4}} .
$$

[28] In the following we consider situations for which $L / \bar{v} \gg D^{c m} / \bar{v}^{2}$ so that the travel time mean and variance can be approximated by

$$
\tau_{L} \approx \frac{L}{\bar{v}}, \quad \sigma_{\tau_{L}}^{2}=2 \frac{L D^{c m}}{\bar{v}^{3}}
$$

For simplicity, in the following, we assume that the control time $T$ is much larger than the standard deviations $\sigma_{\tau L i}(i=$ $b, p)$. Thus, we can approximate the travel time distribution $f\left(L_{i}, t_{i}\right)$ by a delta distribution

$$
f(L, t) \approx \delta\left(t-\frac{L}{\bar{v}}\right)
$$

Substituting (6a)-(6c), (7) and (12) into (4) and (5) yields

$$
\begin{aligned}
P[P 2]= & \frac{1}{2 \pi \sigma_{b} \sigma_{p}} \int_{y_{p_{\min }}}^{y_{p_{\max }}} \int_{-\infty}^{y_{b_{\min }}} \exp \left[-\frac{y_{b}^{2}}{2 \sigma_{b}^{2}}\right] \\
& \times \exp \left[-\frac{\left(y_{p}-y_{b}\right)^{2}}{2 \sigma_{p}^{2}}\right] \mathrm{d} y_{b} \mathrm{~d} y_{p} \\
& +\frac{1}{2 \pi \sigma_{b} \sigma_{p}} \int_{y_{p_{\min }}}^{y_{p_{\max }}} \int_{-\infty}^{y_{b_{\min }}} \exp \left[-\frac{y_{b}^{2}}{2 \sigma_{b}^{2}}\right] \\
& \times \exp \left[-\frac{\left(y_{p}-y_{b}\right)^{2}}{2 \sigma_{p}^{2}}\right] \mathrm{d} y_{b} \mathrm{~d} y_{p},
\end{aligned}
$$

where we define $\sigma_{i}^{2}=2 D^{c m} \tau_{i}$ for $i=b, p$.

[29] It is worthwhile emphasizing that the probability estimate (13) ignores heterogeneity as a source of uncertainty and risk. It can be extended to mildly heterogeneous aquifers (without dominant preferential flow paths) by replacing spatially varying transmissivity $T(\mathbf{x})$ and porosity $\phi(\mathbf{x})$ with their effective values $T_{\text {eff }}$ and $\phi_{\text {eff, }}$, respectively. Then the statistics of macroscopic flow velocity $\mathbf{v}$ can be obtained from those of hydraulic head gradient $\mathbf{J}$ by using Darcy's law $\mathbf{v}=-\left(T_{\text {eff }} / \phi_{\text {eff }}\right) \mathbf{J}$. Expressions for the effective hydraulic properties of heterogeneous aquifers can be found in work by Dagan [1989] and Tartakovsky and Neuman [1998], among others.

[30] Preferential flow paths (e.g., paleochannels) can redirect the plume around the reactive barrier, significantly affecting the probability estimate $P[P 2]$ [e.g., Korte, 2001].The likelihood of the existence of dominant preferential flow paths can be elucidated from expert opinion of the kind we discuss in section 5. In the present analysis we assume that the occurrence of preferential flow paths was deemed unlikely, i.e., that the probability estimate (13) with the generalization described above is valid.

\subsection{Probability of PATH 3}

[31] We define $P[P 3]$ as the probability that the center of mass of the plume enters the protected region $\Omega_{p}$ after having passed through the reactive barrier $\Omega_{b}$. In analogy with $(3 b)$,

$$
P[P 3]=P\left[m_{y}\left(t_{b}\right) \in \Omega_{b} ; m_{y}\left(t_{b}+t_{p}\right) \in \Omega_{p} ; 0<t_{p}+t_{b}<T\right] .
$$


The reasoning presented for the probability of PATH 2 leads to the probability of occurrence of $P 3$ in a mildly heterogeneous aquifer given by

$$
\begin{aligned}
P[P 3]= & \frac{1}{2 \pi \sigma_{b} \sigma_{p}} \int_{y_{p_{\min }}}^{y_{y_{\max }}} \int_{y_{b_{\min }}}^{y_{b_{\max }}} \exp \left[-\frac{y_{b}^{2}}{2 \sigma_{b}^{2}}\right] \\
& \times \exp \left[-\frac{\left(y_{p}-y_{b}\right)^{2}}{2 \sigma_{p}^{2}}\right] \mathrm{d} y_{b} \mathrm{~d} y_{p} .
\end{aligned}
$$

\subsection{Probability of Natural Attenuation Failure}

[32] Natural attenuation (NA) has a potential to reduce contaminant concentration $C$ below the threshold value $C^{*}$ regardless of whether the plume takes PATH 2 or PATH 3. The probability of failure of NA to do so is estimated below for both paths.

\subsection{1. $P[N A]$ Along PATH 2}

[33] The probability of failure of NA of a plume traveling along PATH 2 corresponds to the first minimal cut set in (2),

$$
P[P 2 \cap N A]=P[N A \mid P 2] P[P 2] .
$$

The conditional probability in (16) can be defined as

$$
P[N A \mid P 2]=P\left[C_{\text {peak }}^{P 2}\left(\mathbf{x} \in \Omega_{p}, t \leq T\right)>C^{*} \mid P 2\right],
$$

where $C_{\text {peak }}^{P 2}$ is the maximum concentration, and $C^{*}$ is the concentration threshold value. Assuming a Gaussian shape for the concentration distribution, the peak concentration is given by

$$
C_{\text {peak }}^{P 2}(t)=\frac{C_{0} \exp \left[-\lambda_{N A} t\right]}{4 \pi t \sqrt{D_{L}^{e f f} D_{T}^{e f f}}} .
$$

[34] Here $D_{L}^{\text {eff }}$ and $D_{T}^{\text {eff }}$ are the longitudinal and transverse effective dispersion coefficients, $\lambda_{N A}$ is a natural attenuation degradation coefficient, and $C_{0}$ is an initial concentration. We take $C_{0}$ to be constant and deterministic, but our methodology can be extended to include uncertainty in the initial concentration. It is also worthwhile emphasizing that the methodology presented here is equally applicable to two and three spatial dimensions.

[35] In homogeneous aquifers, the effective dispersion coefficients $D_{L}^{e f}$ and $D_{T}^{e f f}$ coincide with their local counterparts. Heterogeneity complicates the analysis [e.g., Gelhar and Axness, 1983; Dagan, 1988; Kitanidis, 1988; Dentz et $a l ., 2000]$ and appropriate effective dispersion coefficients should be used [e.g., Dagan, 1989]. For short travel distances, the use of the local dispersion coefficients in (18) yields a conservative estimate of the peak concentration.

[36] Since the peak concentration $C_{p e a k}^{P 2}$ in (18) decreases monotonically with time $t$, one can rewrite (17) as

$$
P[N A \mid P 2]=P\left[\mathbf{x} \in \Omega_{p}, t_{\text {peak }}<t^{*} \mid P 2\right],
$$

where $t_{\text {peak }}$ is the arrival time of $C_{\text {peak }}$, and $t^{*}$ is obtained as a solution of the equation

$$
C_{\text {peak }}^{P 2}\left(t^{*}\right)=C^{*}
$$

We quantify the arrival time of the peak concentration by the arrival time distribution of the center of mass (8). It follows from (19) and (9) that

$$
P[N A \mid P 2]=\int_{0}^{t^{*}} f\left(t_{\text {peak }}, L_{P_{2}}\right) \mathrm{d} t_{\text {peak }}=F\left(t^{*}, L_{P_{2}}\right) .
$$

Note that $L_{P 2}$ is some characteristic length of the plume trajectory within $P 2$, whose precise value needs to be selected on the basis of expert opinion. A conservative estimate might be the shortest distance, which would provide for a worst case scenario.

\subsection{2. $P[N A]$ Along PATH 3}

[37] The probability of failure of NA of a plume traveling along PATH 3 corresponds to the second minimal cut set in (2),

$$
P[P 3 \cap R E \cap N A]=P[N A \mid P 3 \cap R E] P[R E \mid P 3] P[P 3] .
$$

The reasoning used in the section above leads to

$$
P[N A \mid R E \cap P 3]=P\left[C_{\text {peak }}^{P 3}\left(\mathbf{x} \in \Omega_{p}, t \leq T\right)>C^{*} \mid R E, P 3\right],
$$

where

$$
C_{\text {peak }}^{P 3}(t)=\frac{C_{0} \exp \left[-\lambda_{N A}\left(t-\Delta t_{R E}\right)-\lambda_{R E} \Delta t_{R E}\right]}{4 \pi t \sqrt{D_{L}^{e f f} D_{T}^{e f f}}} .
$$

[38] Here $\lambda_{R E}$ is a decay coefficient associated with the reactive barrier and $\Delta t_{R E}$ is the residence time of the solute plume moving through the barrier. The latter can be estimated as

$$
\Delta t_{R E}=w / \bar{v}
$$

where $w$ is the width of the reactive barrier in the flow direction. In analogy to (19), (23) and (24) give rise to

$$
P[N A \mid R E \cap P 3]=P\left[x \in \Omega_{p}, t_{\text {peak }}<\hat{t} \mid R E, P 3\right],
$$

where $\hat{t}$ is a solution of

$$
C_{\text {peak }}^{P 3}(\hat{t})=C^{*}
$$

[39] In analogy to (21), we obtain

$$
P[N A \mid R E \cap P 3]=F\left(\hat{t}, L_{P_{3}}\right) .
$$

[40] Note that the characteristic length of the plume trajectory $L_{P 3}$ differs from that of $P 2$. Again, the selection of this property should be based on expert knowledge and a conservative estimate would be the minimum trajectory between the contaminated source and the protection area that circumvents the reactive barrier.

\subsection{Probability of Reactive Barrier Failure}

[41] There are many reasons why a permeable reactive barrier (PRB) might fail (Federal Remediation Technologies 
Roundtable, Evaluation of permeable reactive barrier performance, 2002, available at http://www.clu-in.org/download/ rtdf/2-prbperformance_web.pdf). One of them, failure to intercept a contaminant plume, has been considered above. Others that must be considered include (1) preferential flow paths through the barrier that can cause rapid local depletion of reactive substances [Elder et al., 2002], (2) reactive performance decline where the barrier becomes passive before the entire mass of reactant is used up (ITCR, www.itrcweb. org), (3) microbial growth that can impede flow [e.g., Gavaskar et al., 2002; Wilkin and Puls, 2003; Gu et al., 2002], and (4) tidal fluctuations that can affect performance of reactive barriers in coastal regions [e.g., Ludwig et al., 2002].

[42] Some sources of uncertainty associated with the performance of PRBs can be related mathematically to the uncertainty in hydraulic and reactive properties of PRBs. For example, uncertainty about kinetic reaction rates and/or flow velocity through a barrier can be quantified in a way that provides a full pdf for solute concentration exiting the barrier [Tartakovsky et al., 2009]. Rather than attempting to do so, we employ past performance to estimate the probability of a barrier's failure $P[R E]$, a procedure that allows us to demonstrate how expert knowledge can be incorporated into probabilistic risk analysis.

[43] ITCR (www.itrcweb.org) reviews 200 applications of PRBs. Henderson and Desmond [2007] found sufficient specific public information on field operation conditions and performance issues for 40 of the ITRC 200 cases. They identified three main reason for failure: adverse loss of reactivity ( 1 case), adverse hydraulic changes ( 2 cases) and bad design (37 cases). The data of ITCR (www.itrcweb.org) and Henderson and Desmond [2007] suggest that 25\% of all reported cases where PRBs had performance issues are attributable to event PATH 2, i.e., to a plume or its significant part having missed the reactive barrier. Therefore 30 of the 200 cases can be said to have failed after passing through a reactive barrier. This leads us to estimate the probability of failure as

$$
P[R E \mid P 3]=0.15 \text {. }
$$

[44] A few comments about the veracity of the estimate (29) are in order. First, some authors [e.g., Wilkin and Puls, 2003; Henderson and Desmond, 2007] have argued that both the number of case studies currently available and the relatively short periods of experimentation hamper the assessment of the long-term performance of PRBs, and that more information is needed for more accurate long-term predictions. From a PRA perspective this is acceptable as probabilities used in PRAs are subjective, and one can change the probabilities of failure as more information becomes available. Second, it is worthwhile putting the probability estimate (29) into a broader perspective. While the $15 \%$ probability of failure might seem high, it compares favorably with the reliability of other remediation techniques. For example, a study of pump-and-treat remediation $[N R C, 1994]$ identified that at 69 of the 77 sites the system was deemed to have failed. Finally, since many of the cases where the PRBs can be attributed to bad design, it is expected that this number will decrease significantly with increased knowledge and experience.

\section{Probability of System Failure}

[45] The probability of failure of a permeable reactive barrier remediation effort (2) reads in terms of the previously determined probabilities

$$
\frac{P[S F]}{P[S O]}=P[N A \mid P 2] P[P 2]+P[N A \mid R E \cap P 3] \times P[R E \mid P 3] P[P 3],
$$

where $P[N A \mid P 2]$ given by (21), $P[P 2]$ by (13), $P[N A \mid R E \cap$ $P 3]$ by (28), $P[R E \mid P 3]$ by (29) and $P[P 3]$ by (15). The cumulative travel time distribution $F(t, L)$ of the center of mass positions is given by (9).

[46] This closed-form analytical solution provides a probabilistic description of a complex reactive transport phenomenon and can be used to analyze rare events. This is in contrast with a vast majority of existing stochastic analyses, including Monte Carlo simulations, which predict the mean behavior of a system and quantify predictive uncertainty through corresponding (co)variances. PRA facilitates the uncertainty quantification by taking a system's approach in which a complex system with many uncertain parameters is subdivided into a set of constitutive components (basic events), each of which has fewer parameters.

[47] The derivation of the analytical estimate (30) of the probability of failure of a remediation effort required some level of abstraction. This estimate can be refined by conducting numerical analyses of the system's components. Numerical tools one would employ for such analyses (Monte Carlo simulations, particle tracking, etc.) become much more tractable because of significant reductions in the number of uncertain parameters and degrees of freedom.

\section{Sensitivity Analysis}

[48] The probability of remediation effort failure (30) depends on a number of geometrical (the length of a reactive barrier $2 \mathrm{~W}$, the distance between the source of contamination and the barrier $L_{b}$, etc.) and the statistical (mean, variance, and correlation of temporal fluctuations of hydraulic head gradient, etc.) parameters, as well as on effective hydraulic and transport properties (effective hydraulic conductivity, porosity, dispersion coefficients, etc.). To discern the influence of these parameters on risk assessment, we conduct a sensitivity analysis. We start by normalizing geometric characteristics with $L$, the distance from the source of contamination to the protected region $\left(L=L_{b}+L_{p}\right)$, and time with the correlation time $\lambda_{v}$. Some of the key dimensionless quantities arising from such a normalization are

$$
\begin{gathered}
\kappa=\frac{\bar{v} \tau_{v}}{L}, \quad \alpha=\frac{L_{b}}{L}, \quad t_{d}^{*}=\frac{t^{*}}{\tau_{v}}, \quad \hat{t}_{d}=\frac{t^{* *}}{\tau_{v}} \\
l_{P 2}^{*}=\frac{L_{P 2}}{L}, \quad l_{P 3}^{*}=\frac{L_{P 3}}{L}, \quad \hat{w}=\frac{W}{L} .
\end{gathered}
$$

[49] We set the coordinate system $y=0$ at the center of the reaction barrier. If the barrier's dimensionless length is 


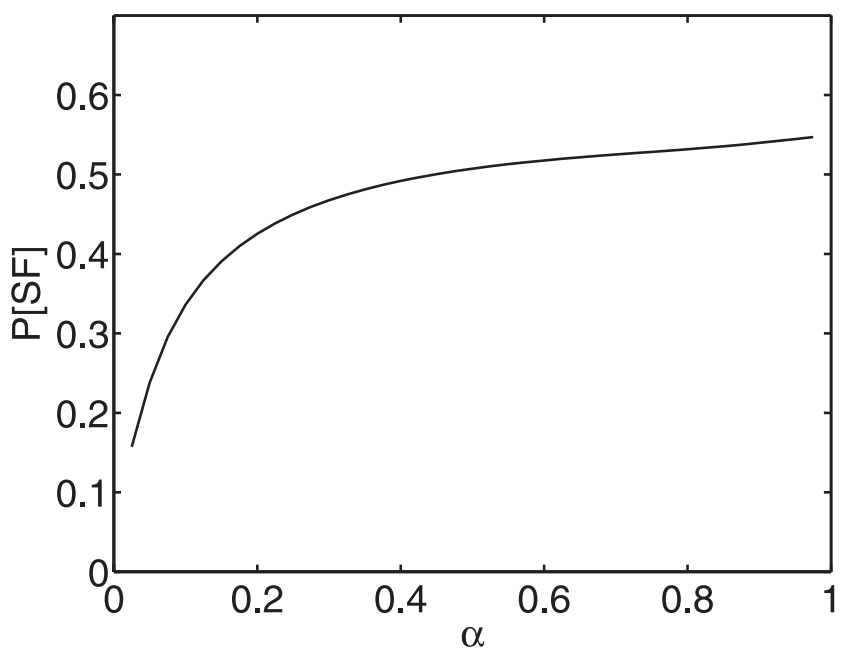

Figure 4. Dependence of probability of failure of the remediation effort on the distance between the source of contamination and the reactive barrier.

$2 \hat{w}$, this gives $y_{b_{\min }}=-\hat{w}$ and $y_{b_{\max }}=\hat{w}$ and sets the mean (expected) locations of the center of mass to $\bar{m}_{b}=0$ and $\bar{m}_{p}=0$.

[50] To investigate the influence of various parameters on the probability of failure we set $\sigma_{v}=1, \kappa=0.1, \alpha=0.5, \hat{w}=$ $0.125, y_{2 \min }=-0.5, y_{2 \max }=0.5, t^{*}{ }_{d}=100, \hat{t}_{d}=100, l_{P 2}=$ 1.1 , and $l_{P 3}=1$, and then vary one parameter at the time.

[51] The probability of failure of the remediation effort increases monotonically with $\alpha$, the relative distance between the reactive barrier and the source of contamination (Figure 4). This reflects the fact that the larger the distance a contaminant plume travels before reaching the plane where the barrier is located, the greater the likelihood of the plume bypassing the reactive barrier.

[52] Figure 5 shows that the probability of remediation failure decreases with the barrier's length $2 \hat{w}$. Such a decrease is, of course, to be expected as wider barriers are more likely to intercept a plume. The added value of this calculation is that it quantifies the risk, thus facilitating decision making (e.g., a cost benefit analysis). For example, one can see that increasing the barrier's length beyond $\hat{w}=$ 0.5 does not really reduce the probability of remediation failure, making the added expense unnecessary.

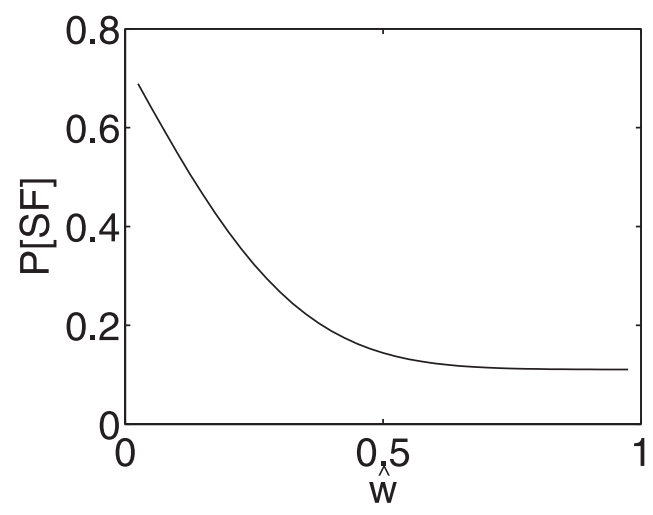

Figure 5. Dependence of probability of failure of the remediation effort on the length of the reactive barrier.

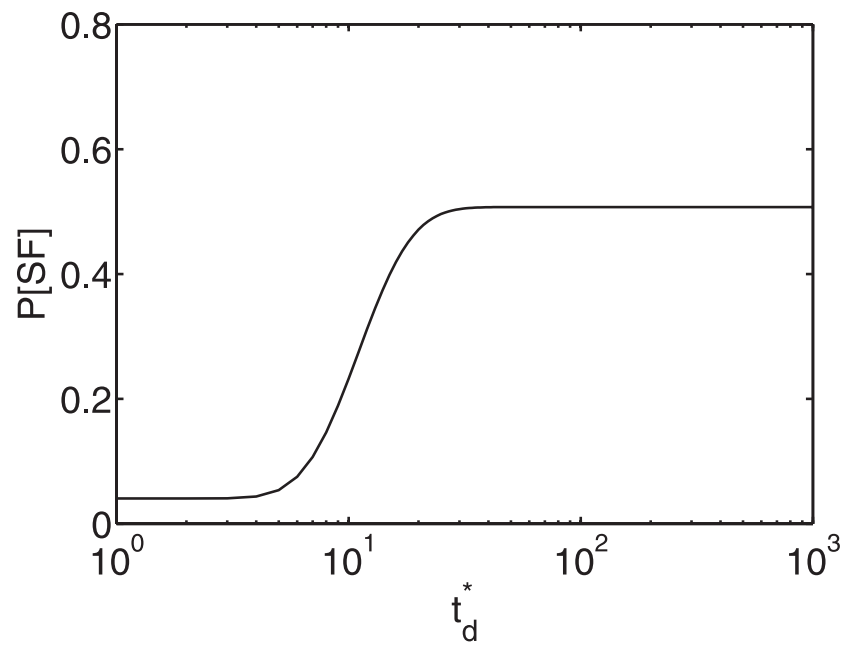

Figure 6. Dependence of probability of failure of the remediation effort on the normalized time $\hat{t}^{*}$ that is required for natural attenuation to succeed.

[53] Figure 6 reveals a nearly step-like dependence of the probability of remediation failure on the (normalized) time $t$ 当 it takes natural attenuation to reduce contaminant concentration to acceptable levels. For $t_{d}^{*}$ smaller than some critical value $\left(t_{c r} \approx 10\right.$ for the parameters used in Figure 6 ) natural attenuation alone is sufficient, contamination will be unlikely to occur regardless of whether a reactive barrier is operational, so that $P[S F]=0.04$. For $t_{d}^{*}>t_{c r}$, the probability of remediation failure rapidly approaches its maximum value $(P[S F] \approx 0.51$ for the parameters used in Figure 6). For small $t$, failure can still occur if the plume arrives faster than this time, which is determined by the statistics of the velocity field. At larger $t$, it depends on the probability that the plume misses the protection zone or that the reactive barrier is successful. This suggests that a detailed assessment of the natural attenuation time is not necessary as long as one can be reasonably sure that $t_{d} \ll t_{c t}$ or $t_{d}^{*} \gg t_{c t}$. The dependance of probability of failure on $\hat{t}_{d}$ is similar.

[54] Figures 7 and 8 elucidate the degree to which uncertainty in temporal fluctuations of hydraulic head gradient and, hence, macroscopic velocity affect the probability of remediation failure. Large temporal fluctuations, as quantified by high dimensionless variance $\hat{\sigma}$ (Figure 7) and/or small dimensionless correlation length (the Kubo number $\kappa$ ) (Figure 8), increase the likelihood that the plume will bypass the protected zone $\Omega_{p}$, thus reducing the probability of failure. Small temporal fluctuations likewise reduce the probability of failure, but through a different mechanism. This mechanism is that either small variances or long correlations ensure that the plume does not deviate much from its mean trajectory, increasing its chances of being intercepted by the reactive barrier. Thus, because of these two mechanisms the probability of failure first increases, peaks and then decreases between these two extreme regimes.

[55] Finally, dependence of the probability of remediation effort failure on $l_{P 3}$, the normalized distance a plume that bypassed the remediation barrier has to travel before it reaches the protected zone, is shown in Figure 9. The 


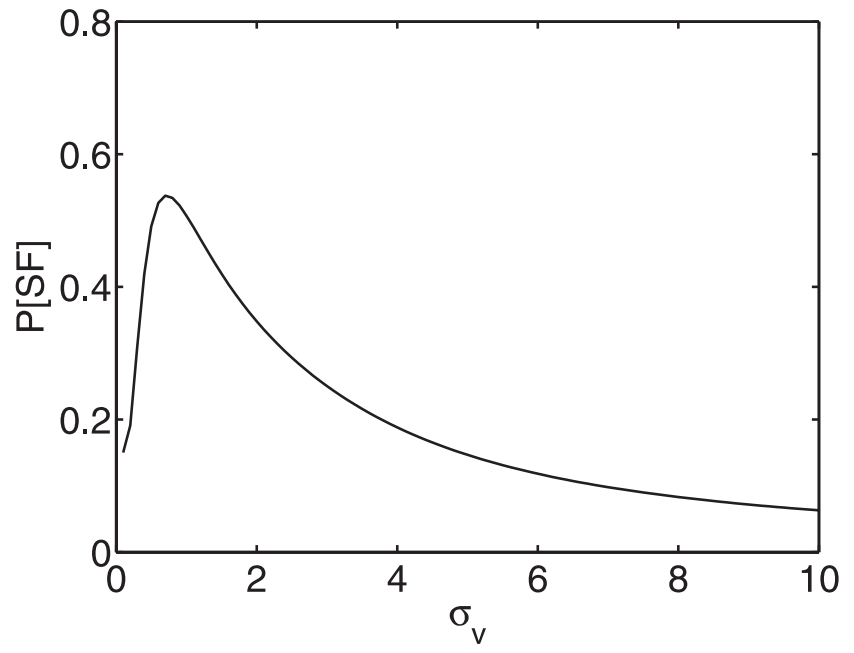

Figure 7. Dependence of probability of failure of the remediation effort on the uncertainty about temporal fluctuations of mean hydraulic gradient, as quantified by standard deviation of velocity $\sigma_{v}$.

behavior is analogous to that in Figure 6 as the probability of failure rapidly drops from one asymptote to another once the distance traveled is greater than some critical value. The behavior for $l_{P 2}$ is similar.

\section{Summary and Discussion}

[56] We used a rigorous probabilistic risk assessment (PRA) to estimate the probability of the potential failure of a permeable reactive barrier to prevent contamination of a protected region located down gradient from a contaminant plume. (Other remediation techniques can be analyzed in a similar manner.) The methodology consists of defining a fault tree specifying all the potential options that can lead to failure of a specified remediation techniques. Then the probability of remediation failure can be broken into individual probabilities (either conditional or unconditional) that are combined through Boolean operators. Our analysis leads to the following major conclusions.

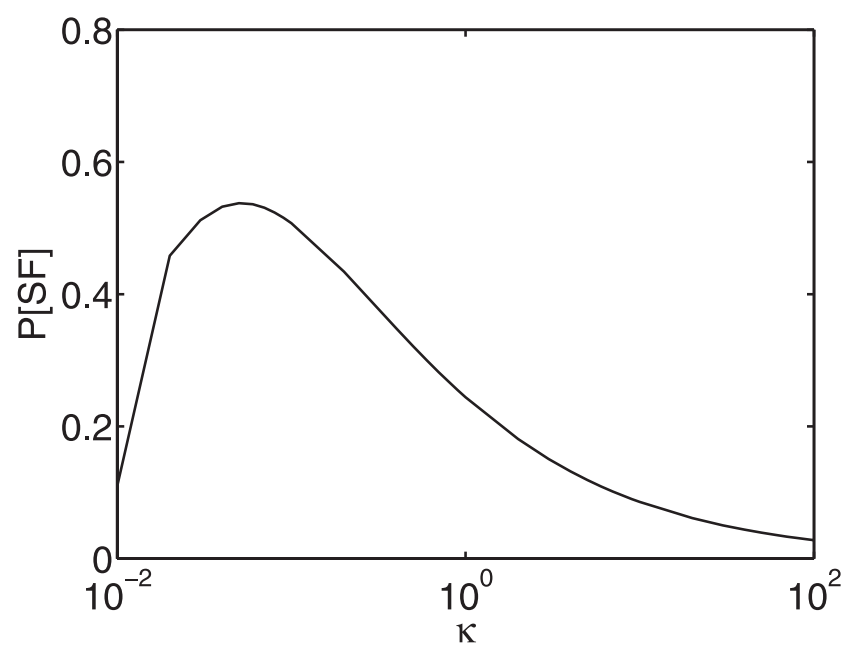

Figure 8. Dependence of probability of failure of the remediation effort on the Kubo number $\kappa$.

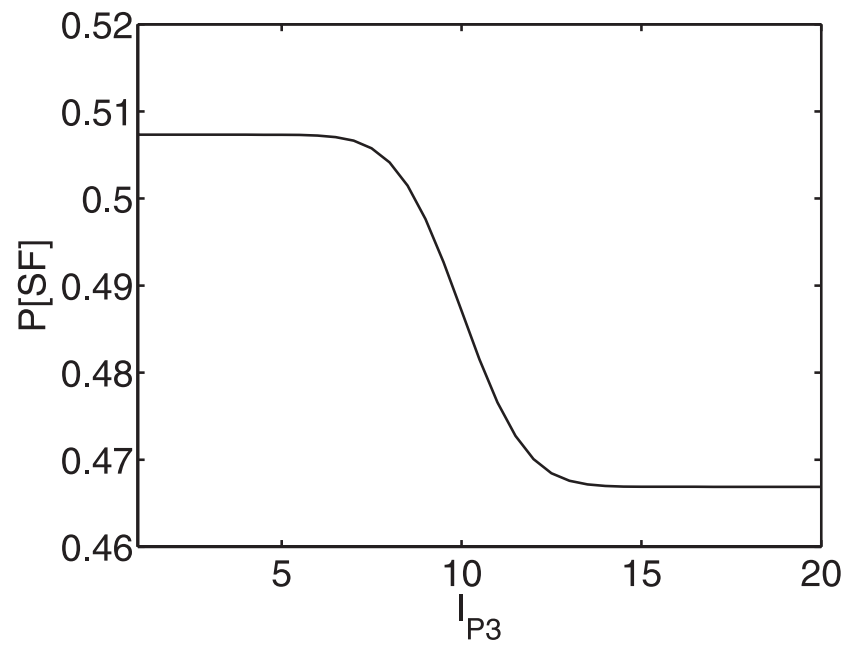

Figure 9. Dependence of probability of failure of the remediation effort on the normalized distance a plume that bypassed the remediation barrier has to travel before it reaches the protected zone.

[57] 1. PRA provides a probabilistic description of a complex reactive transport phenomenon and can be used to analyze rare events. This is in contrast with a vast majority of existing stochastic analyses, including Monte Carlo simulations, which predict the mean behavior of a system and quantify predictive uncertainty through corresponding (co)variances.

[58] 2. PRA facilitates uncertainty quantification by taking a system's approach in which a complex system with many uncertain parameters is subdivided into a set of constitutive components (basic events), each of which has fewer parameters.

[59] 3. The derivation of our analytical estimate of the probability of failure of a remediation effort required some level of abstraction. This estimate can be refined by conducting numerical analyses of the system's components. Numerical tools one would employ for such analyses (Monte Carlo simulations, particle tracking, etc.), become much more tractable because of significant reductions in the number of uncertain parameters and degrees of freedom.

[60] In addition to operational advantages, PRA acts as a translator of information between scientists, engineers, investors, politicians and decision makers. Each expert can work within their area of expertise and work on their "branch" of the problem without having to understand the complexities of the whole problem. The results of such an approach can then be used in the context of a decision support system (DSS) [Bedford and Cooke, 2003] that uses decision theory [Berger, 1980; Anand, 2002] to aid proper decision making. More importantly, a probabilistic risk assessment poses directly the problem in an uncertain perspective, which allows it to account for uncertainties inherent to subsurface environments.

[61] The presented PRA can be further refined by accounting for additional sources of uncertainty, including the existence of several competing conceptual models, spatial heterogeneity of hydraulic parameters and the spatial distribution of reactive material within the barrier itself. Such extensions would require additional advances in stochastic analysis of subsurface flow and transport, as well as in data assimilations. 
[62] Acknowledgments. This study originated at a workshop/class taught by D.M.T. at the Technical University of Catalonia. It was sponsored by the Generalitat de Catalunya. D.B. would like to acknowledge the financial support of the Spanish Ministry of Science via the "Juan de la Cierva" program. The work of D.M.T. was supported by the Office of Science of the U.S. Department of Energy, Advanced Scientific Computing Research (ASCR) program in Applied Mathematical Sciences.

\section{References}

Alvarez, P., and W. Illman (2006), Bioremediation and Natural Attenuation, John Wiley, Hoboken, N. J.

Anand, P. (2002), Foundations of Rational Choice Under Risk, Oxford Univ. Press, Oxford, U. K.

Batchelor, B., J. Valdes, and V. Araganth (1998), Stochastic risk assessment of sites contaminated by hazardous wastes, J. Environ. Eng., 124, $380-$ 388.

Bedford, T., and R. Cooke (2003), Probabilistic Risk Analysis: Foundations and Methods, Cambridge Univ. Press, Cambridge, U. K.

Berger, J. (1980), Statistical Decision Theory and Bayesian Analysis, Springer, New York.

Bolster, D., and D. M. Tartakovsky (2008), Probabilistic risk analysis of building contamination, Indoor Air, 15(5), 351-364, doi:10.1111/j.16000668.2008.00536.x.

Dagan, G. (1988), Time-dependent macrodispersion for solute transport in anisotropic heterogeneous aquifers, Water Resour. Res., 24(9), 14911500 .

Dagan, G. (1989), Flow and Transport in Porous Formations, Springer, New York.

Dentz, M., and J. Carrera (2003), Effective dispersion in temporally fluctuating flow through a heterogeneous medium, Phys. Rev. E, 68, 36310

Dentz, M., H. Kinzelbach, S. Attinger, and W. Kinzelbach (2000), Temporal behavior of a solute cloud in a heterogeneous porous medium: 1 . Point-like injection, Water Resour. Res., 36(12), 3591-3604.

Elder, C. R., C. H. Benson, and G. R. Eykholt (2002), Effects of heterogeneity on influent and effluent concentrations from horizontal permeable reactive barriers, Water Resour. Res., 38(8), 1152, doi:10.1029/ 2001WR001259.

Garrick, B. J. (1989), Risk assesment practices in the space industry: The move towards quantification, Risk Anal., 9, 1-7.

Gavaskar, A. B., N. Sass, E. Drescher, W. Yoon, J. Sminchak, J. Hicks, and W. Condit (2002), Evaluating the longevity and hydraulic performance of permeable barriers at Department of Defense sites, technical report, Battelle for Nav. Facil. Eng. Serv. Cent., Columbus, Ohio.

Gelhar, L. W., and C. L. Axness (1983), Three-dimensional stochastic analysis of macrodispersion in aquifers, Water Resour. Res., 19(1), $161-180$.

Gu, B. D., D. Awtson, L. Wu, D. Phillips, D. White, and J. Zhou (2002), Microbiological characterization in a zero-valent-iron reactive barrier, Environ. Monit. Assess., 77, 293-307.

Henderson, A., and A. Desmond (2007), Long-term performance of zerovalent iron permeable reactive barriers: A critical review, Environ. Eng Sci., 24, 401-423.

Kitanidis, P. K. (1988), Prediction by the method of moments of transport in heterogeneous formations, J. Hydrol., 102, 453-473.

Korte, N. (2001), Zero-valent iron permeable reactive barriers: A review of performance, Rep. 5056, Environ. Sci. Div., U.S. Dep. of Energy, Washington, D. C.
Ludwig, R. D., R. G. McGregor, D. W. Blowes, S. G. Benner, and K. Mountjoy (2002), A permeable reactive barrier for treatment of heavy metals, Groundwater, 40, 59-66.

National Research Council (NRC) (1983), PRA procedures guide, Tech. Rep. NUREG/CR-2300, Washington, D. C.

National Research Council (NRC) (1994), Alternatives for Ground Water Cleanup, Natl. Acad., Washington, D. C.

National Research Council (NRC) (1997), Review of recommendations for probabilistic seismic hazard analysis: Guidance on uncertainty and use of experts, technical report, Washington, D. C.

Paté-Cornell, E., and R. Dillon (2001), Probabilistic risk analysis for the NASA space shuttle: A brief history and current work, Reliab. Eng. Syst. Safety, 74(3), 345-352.

Riva, M., X. Sanchez-Vila, A. Guadagnini, M. D. Simoni, and M. Willmann (2006), Travel time and trajectory moments of conservative solutes in twodimensional convergent flows, J. Contam. Hydrol., 82(1-2), 23-43.

Sanchez-Vila, X., and A. Guadagnini (2005), Travel time and trajectory moments of conservative solutes in three dimensional heterogeneous porous media under mean uniform flow, Adv. Water Resour., 28(5), $429-439$.

Silliman, S. E., and C. Frost (1998), Monitoring hydraulic gradient using three-point estimator, J. Environ. Eng., 124(6), 517-523.

Tartakovsky, D. M. (2007), Probabilistic risk analysis in subsurface hydrology, Geophys. Res. Lett., 34, L05404, doi:10.1029/2007GL029245.

Tartakovsky, D. M., and S. P. Neuman (1998), Transient effective hydraulic conductivities under slowly and rapidly varying mean gradients in bounded three-dimensional random media, Water Resour. Res., 34(1), $21-32$.

Tartakovsky, D. M., M. Dentz, and P. C. Lichtner (2009), Probability density functions for advective-reactive transport in porous media with uncertain reaction rates, Water Resour. Res., doi:10.1029/2008WR007383, in press.

U.S. Environmental Protection Agency (1992), Guidelines for exposure assessment, USEPA 600Z-92/001, Washington, D. C.

Vesely, W. E., F. F. Goldberg, N. H. Roberts, and D. F. Haasi (1981), Fault tree handbook, Tech. Rep. NUREG 0492, U.S. Nucl. Regul. Comm., Washington, D. C.

Wang, T., and W. F. McTernan (2002), The development and application of a multilevel decision analysis model for the remediation of contaminated groundwater under uncertainty, J. Environ. Manage., 64, 221-235.

Wilkin, R. T., and R. W. Puls (2003), Capstone report on the application, monitoring, and performance of permeable reactive barriers for groundwater remediation: Vol. 1. Performance evaluation at two sites, techical report, U.S. Environ. Protect. Agency, Washington, D. C.

Winter, C. L., and D. M. Tartakovsky (2008), A reduced complexity model for probabilistic risk assessment of groundwater contamination, Water Resour. Res., 44, W06501, doi:10.1029/2007WR006599.

M. Barahona, D. Bolster, M. Dentz, D. Fernandez-Garcia, P. Trinchero, X. Sanchez-Vila, and C. Valhondo, Department of Geotechnical Engineering and Geosciences, Technical University of Catalonia, E-08034 Barcelona, Spain. (xavier.sanchez-vila@upc.edu)

D. M. Tartakovsky, Department of Mechanical and Aerospace Engineering, University of California, San Diego, La Jolla, CA 92093, USA. (dmt@ucsd.edu) 